\title{
Why send probes to Venus?
}

\section{Professor Donald Hunter of the University of Arizona tells what next week's Venus launch may achieve}

THE Pioneer Venus Orbiter is well on its way, after a perfect launch on 20 May. If all goes well, early on the morning of 7 August it wil be followed by the Multiprobe. These are actually two separate missions, telescoped together by the mundane realities of obtaining funds. Originally, they were supposed to go at successive opportunities, but various budgetary delays caused them to be flown the same year. The Orbiter takes a longer and somewhat slower path; both arrive in early December, about five days apart.

Venus and Earth seem as if they should be twins; their mass and radius are almost identical; although Venus gets twice the solar flux, it actually absorbs nearly the same amount of heat because it reflects a larger fraction. Instead, on Venus, we find a planetwide cloud of $\mathrm{H}_{2} \mathrm{SO}_{4}$ droplets and a uniform surface temperature of about $750 \mathrm{~K}$ at the bottom of $90 \mathrm{~atm}$ of $\mathrm{CO}_{2}$. These ingredients also exist on Earth, but the $\mathrm{CO}_{2}$ is nearly all buried as limestone, and the sulphur too is mostly buried. $\mathrm{HCl}$ in the stratosphere suppresses all detectable signs of an ozone layer. Many of. these differences are traceable to the presence or absence of liquid water: it seems that Venus either never had much, or somehow lost it. The other differences are reminiscent of what we are now doing to our own planet-releasing large amounts of $\mathrm{SO}_{2}$ from industrial processes; building up the $\mathrm{CO}$. level of the atmosphere through combustion of fossil fuels; and releasing chlorinated hydrocarbons at an ever-increasing rate. In truth, we are not likely to turn Earth into another Venus, but a better knowledge of Venus should give us a better chance to assess the consequences of our current actions.

The ideas are examples of comparative planetology applied to practical concerns. There are many additional areas whose interest is mainly intellectual, for example:

- Study of the upper atmosphere and the ionosphere and their effect of and on the solar wind (similar things may happen occasionally on Earth when its protective magnetic field is temporarily absent during a reversal.)

- The meteorology of a planet very different from our own. Venus rotates very slowly, and atmospheric motions are also slow, except near the cloud tops where there is a steady $100 \mathrm{~m} / \mathrm{s}$ wind in the same retrograde direction as the rotation. mapper on the Orbiter.
- Mapping of surface features to permit study of geological processes.

How do the Pioneer Venus missions contribute to these fields and how do they fit into the (presumably) continuing program of the USSR? The latter question can only be answered in retrospect, since the payloads are known only for past missions. However, the main emphasis of recent Soviet studies has been on surface measurements, whereas Pioneer Venus concentrates on the atmosphere, apart from the radar

The Pioneer Venus Multiprobe mission consists of 5 separate vehicles which travel together to the vicinity of Venus. The 4 probes enter at widely separate points and form a miniature meteorological network; they each measure pressure, temperature, acceleration, and cloudiness and are accurately tracked from Earth to give wind profiles. The bus, in entering the upper atmosphere. measures the neutral and positive-ion composition to supplement the data from the Orbiter. One of the probes is considerably larger than the other three and carries additional instruments to measure composition (mass spectrometer and gas chromatograph), radiation fluxes. and cloud particle properties. Though there are many particular objectives, a general theme is the meteorology and energy balance of the atmosphere below the clouds: how the atmosphere moves and the surface stays hot.

The Orbiter carries 12 experiments. two of which study the meteorology and energy balance in visible and thermal infrared radiation. The local, detailed views from the probes are thus taken to the global scale. There are 5 aeronomy instruments that measure the upper atmosphere and ionosphere directly during the lowest

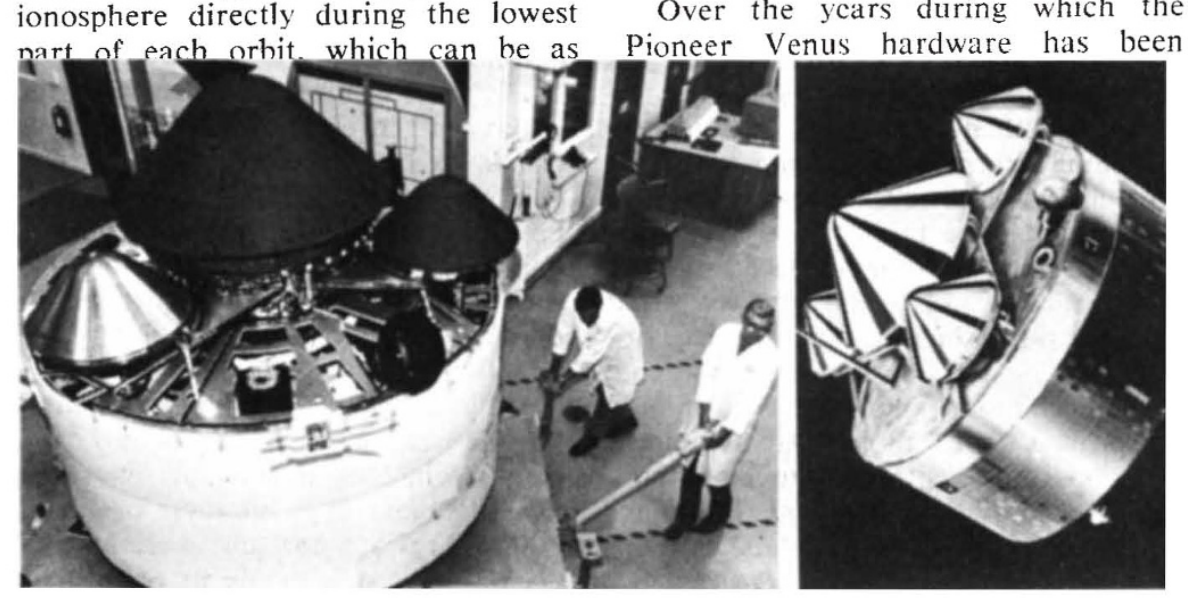

Pioneer's Multiprobe: test model and artist's impression (right).

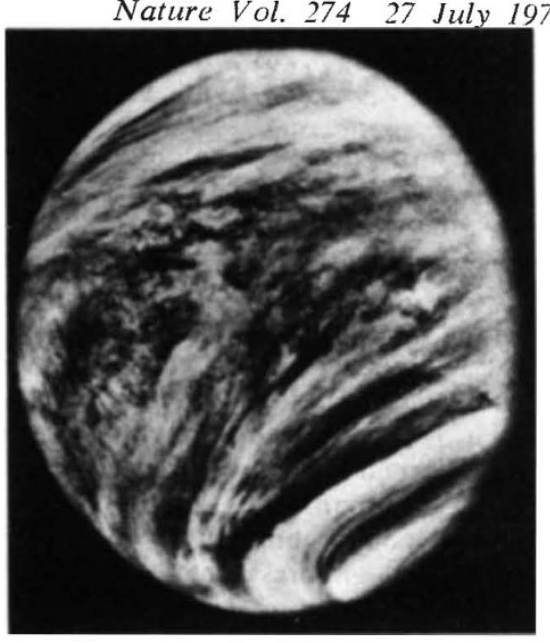

Ultraviolet picture of Venus from Mariner 10, 450,000 miles up.

close as $150 \mathrm{~km}$ to the surface. Additional information comes from radio occultation measurements and the deceleration of the spacecraft due to the atmospheric drag. Three more instruments measure the fields and plasma streaming by from the Sun. All 8 taken together should tell a great deal about the interaction of the two media. The radar will give a low-resolution reflectivity map of one hemisphere along with altimetry of the track below the spacecraft. Information on the interior can be obtained from gravity data and any internal magnetic field that may turn up. Finally, the Orbiter carries a gammaray burst detector; the long Earth-Venus baseline gives excellent direction-finding abilities for these mysterious events. The Orbiter will make one revolution per day for a full Venus year (243 days), and could continue even longer if it is still working.

Though Pioneer Venus is a NASA mission, managed by its Ames Research Center and built by the Hughes Aircraft Company, two of the aeronomy instruments are supplied by the Federal Republic of Germany. and a French scientist participates in the cloud-measuring experiments.

Over the ycars during which the Pioneer Venus hardware has been

(C) Macmillan Journals Ltd 1978 
planned and built, much has been learned about the planet, but there are still plenty of questions. The Soviet Veneras 5 and 6 (1969) extended the chemical analysis of the atmosphere that had begun two years earlier with Venera 4 and Mariner 5. Venera 7 (1970) finally reached the surface and confirmed the temperature and radius deduced less directly by radio and radar. Venera 8 (1972) showed that some sunlight penetrates to the surface, and measured the natural radioactivity of the rocks. Mariner 10, on its way by to Mercury, took the famous photographs of cloud structures (left), confirming the $100 \mathrm{~m} / \mathrm{s}$ winds, and made measurements of the upper atmosphere. Veneras 9 and 10 (1975) deployed probes and orbiters. Each probe took a panoramic picture of the nearby surface and made other measurements of surface properties.

On the way down, the probes showed that the clouds extend from 65 down to $40 \mathrm{~km}$, and confirmed the Earth-based deductions that the principal particles are $\mathrm{H}_{2} \mathrm{SO}_{4}$, about $1 \mu \mathrm{m}$ radius. A mass spectrum of the atmosphere was also obtained. Orbiter data recently published suggest that the upper boundary of the ionosphere has been traced from a low height (around $270 \mathrm{~km}$ ) in the subsolar region to a much higher value (over $600 \mathrm{~km}$ ) near the terminator. It is possible that similar missions may be launched in August at about the same time as the Pioneer Venus Microprobe.

It may seem strange that the Viking of two years ago is not being followed by further exploration of Mars. Much of the explanation lies in the very long times that are needed to plan and execute a planetary mission. A followon to Viking would have had to be started at least six years ago, when construction of Viking had barely started. Even now, we have not fully assimilated the Viking results, and the form of the next mission is not clear. The activities that led to Pioneer Venus started in 1966, before the encounters of Venera 4 and Mariner 5. Getting it into the NASA budget and through the Congress took from 1970 to 1974 , and hardware activities have been going on ever since. Planetary explorers must take the long view. The United States will return to Mars some day, and even to the Moon and Mercury. In the meantime, the Pioneers are going to Venus, and an earlier one will soon encounter Saturn; the Voyagers are on their way to Jupiter, Saturn, and perhaps Uranus; and Galileo is starting its long hardware phase, for a launch to Jupiter in 1982. The waits are long, but the excitement of exploration makes it worthwhile in the end.

\section{Out of the frying pan}

THE English call it mince-meat; the Americans ground beef. But, made into patties, and grilled, barbecued or fried, it is known worldwide as hamburger. It was named for the great Hanse town at the mouth of the Elbe. Unfortunately, there is no city named Cheeseburg, and, although a cheeseburger contains cheese, a hamburger certainly does not contain ham. Possibly the most misbegotten descendant of the hamburger is the nutburger, a culinary and etymological horror that was spawned, of course, in southern California.

When animal or vegetable materials are charred or pyrolysed, carcinogenic derivatives of polycyclic hydrocarbons are produced. Our pithecanthropine ancestors must have dosed themselves with these chemicals as, crouched in caves, they breathed wood smoke and gnawed on burned meat. Their descendants gather around barbecue fires to inhale cigarette smoke while they watch the fat drip from steaks and chops onto red-hot coals from which the smoky flames arise to deposit benzopyrenes on the victuals. The carcinogenic effect of the benzopyrenes may be enhanced by alcohol in beverages that are quaffed in anticipation of the feast.

Last year, Sugimura reported that mutagens were produced when fresh whole fish and beef meat were broiled on steel mesh over a gas flame or charcoal. The smoke condensates from similarly-treated L-trytophan yielded gamma-carboline derivatives that were very potent mutagens. This may mean that tryptophan in meat is the origin of some of the mutagens formed by pyrolysis. The key word "hamburger" was not used in describing the results, and the attention of the newspapers was apparently not aroused. But the fat was in the fire when a group at Washington University, St. Louis, announced, with an accompanying press release, that well-done pan-fried hamburgers contained mutagens that were not present in uncooked, lean, ground beef. The authors recommended cooking "in a microwave oven or under the element of an electrically-heated broiling oven" to avoid formation of mutagens. From the public response to this news it seemed that hamburgers are more famous and have more friends than even apple pie. A newspaper columnist, William Raspberry, said: "When danger waits at every hand, the warnings no longer lead us to safety. They lead us only to anxiety.

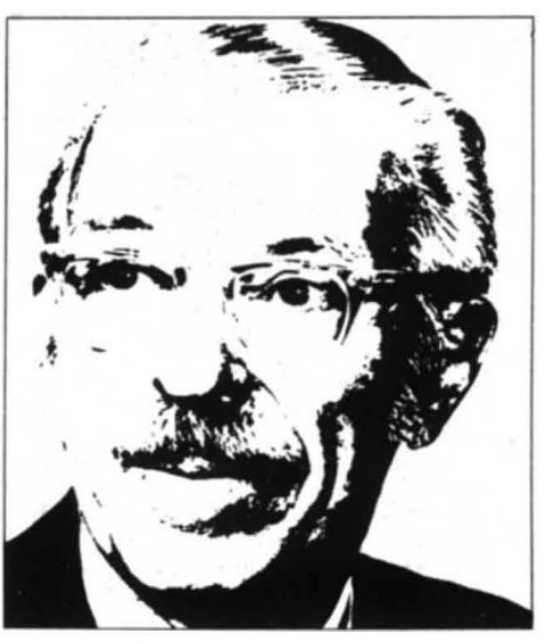

THOMAS H. JUKES

When nearly everything is reported as dangerous, we are forced to behave as though nothing is dangerous".

Carol Tucker Foreman, US Food and Consumer Services Secretary, is well-known for her efforts to protect consumers from hazards of nitrites in bacon. I wonder how this risk compares with leaving hamburgers in the frying pan for an extra minute. I also wonder how to equate the danger caused by less than two nanograms of the estrogen diethylstilbestrol in a meat patty against the effects of what happens when the same meat receives a baptism of fire. Oncologists often declare that no-one knows how much or how little of a carcinogen will produce cancer. Raspberry said: "It would help us if scientists would tell us which substances are more dangerous than others, or in what levels these dangerous substances can be treated as benign. But they won't tell us these things; they say they don't know". It may be time to try and make some educated guesses. Incidentally, Sugimura noted a finding by Mizusaki et al. that the mutagenic activity of the tar derived from tobacco is proportional to the protein content of the tobacco leaves.

We recently were told that fat in the meat we eat increases the risk of cancer of the colon, and that hamburgers should therefore be thoroughly cooked so as to remove most of the fat. Unless the cooking process is carefully controlled, the choice between rare and well-done meat seems a dismal one. We await the next news from the carcinogenologists. 